\title{
Exotic Stars as Challenges to Evolution: an Evaluation
}

\author{
C. Simon Jeffery \\ Armagh Observatory, College Hill, Armagh BT61 9DG, Northern \\ Ireland
}

\begin{abstract}
A star is exotic because, in some way, it extends our knowledge of how stars behave. As we make new observations we discover new phenomena that we do not understand. As we approach an understanding, those phenomena seem less exotic. That is nature. IAU Colloquium 187 set out to discover how far our knowledge of stars now reaches. This is a personal commentary on the proceedings.
\end{abstract}

\section{Introduction}

The IAU Colloquium 187 brought together a multidisciplinary, multiwavelength school of astronomers working on a highly disparate range of objects. In blunt terms, we address the question, "Was the conference worthwhile, and what have we learnt?" The first specific question to consider is, "What makes a star exotic?"

A sign outside the conference hotel, situated on route A1A a few kilometers north of Miami, suggested a possible source of information: "The A1A bar where the stars hang out all night." Since the venue remained securely closed all week, delegates were unable to pursue their researches in that direction, but themselves provided more tangible suggestions. Milone suggested that the question was "not whether a star is exotic or not, but whether it is perceived to be exotic." Knapp refined this observation, citing Trimble: "objects (or people) are only normal when you don't know them very well." Of course, there has to be an exception, and Tout reported of one object that "the more we know about this star the more normal it gets."

However, for all of the remarkable stars discussed, there was not one mention of a star which, because we can observe it in such detail, is perhaps the most exotic of them all. Were one to claim for a point source the surface structure and activity exhibited by the Sun, we would indeed have found an exotic beast. That the Galaxy is most certainly full of such objects in both variety and profusion simply underlines the relevance and timeliness of this conference. In the following summary, I offer a personal perspective of the papers presented, together with some pointers to future work. ${ }^{1}$

\footnotetext{
${ }^{1} \mathrm{~A}$ conference evaluation is obliged, broadly speaking, to acknowledge the contributions of all of the participants, to evaluate, in some sense, what we have learnt, to congratulate and encourage the contributors, and to be brief. Any speaker finally agreeing (or being coerced) to give such
} 


\section{The stars themselves}

As the objects of intense scrutiny, unusual scientific specimens often adopt individual persona. This is just as true of stars. Because the objects are diverse, there is also a need to categorise. The following somewhat anthropomorphic classification echoes genuine intimacies expressed in our conference.

- Familiar Faces. The brightest stars in the sky have frequently provided some of the toughest puzzles for astronomers. Examples include $\beta$ Lyr, $\gamma$ Cas, $\epsilon$ Aur, $\eta$ Car, Sirius, Vega and more. For many of these stars, the wealth of data available to today's astronomer is staggering, yet the puzzles seem no nearer to a solution. This sense of frustration was conveyed by several delegates: Zwitter asked how we might avoid the equation: more data $=$ less understanding. Harmanec suggested that "too many things are known about $[\gamma$ Cas]," while Smith was more sanguine about the fact that "people tend to argue a lot about $\eta$ Car." Over thirty years after their first encounters with HR 2142 and V471 Tau, respectively, two delegates found themselves still trying to solve career-long questions, while Guinan - clearly in for the long haul - found that after 2150 years of observing $\epsilon$ Aur, astronomers still find it puzzling.

- Double Trouble. Binary star systems, by introducing an absolute minimum of two additional free parameters (secondary mass and orbital separation), inevitably give rise to a wider range of problems. Their importance was emphasized by Ivanova who reminded us that binary frequency was in the range $50-150 \%$. Eggleton demonstrated how binary-star evolution has given rise to inexplicable cases such as V379 Cep, OW Gem and V643 Ori. Part of the difficulty lies in keeping track of which star was originally more massive.

Other characters in the cast included RT Lac - "not an exotic star, but a member of an exotic club" (Milone), TU Mus - "exotic because it is so remarkably well behaved" (Terrell) and $\zeta$ Aur - in which circumstellar gas is probed by the hot companion (Griffin).

More problematic are those binary stars in which the companion is unseen and which included HR 2142 (Peters), V505 Mon and companions (Chochol) and the sdB stars (Han). In the case of $\eta$ Car, a putative unseen companion allows a wide range of possible solutions, but in this case critics still argue against the permissiveness of such a "free parameter heaven" (Smith). The discussion of GR Tau (Yamasaki), AH Cnc (Branly) and others remind us that our conference barely skimmed the surface of binary star evolution.

- Three's a Crowd, ... Stars don't just come in pairs; a sizable fraction are members of triple systems. Indeed, Eggleton would argue that "if you can't understand a binary, it's probably a triple." This approach was shown to

a talk anticipates a low score against such benchmarks. Therefore this summary comes with an apology to those who have been misrepresented, undervalued or forgotten. 
be true in several cases including SuWt 2 (Bond), AS Cam (Van Hamme), $\delta$ Ori A (Harvin), and V471 Tau (several authors).

- ... More's a Factory. Emphasizing the point that unusual stars have a higher probability of production in multiple systems, the conference was reminded how star clusters provide a fertile breeding ground for low-mass X-ray binaries, millisecond pulsars, blue stragglers and subdwarf B stars, amongst others (Hurley).

- Stars that go "Bump" in the Night. Up to this point, stellar evolution has really only considered stars that either lose or gain mass quasi-statically during relatively long-lived episodes. In recent years there has been increasing evidence for catastrophic collisions and mergers, both between young massive stars (Ivanova) and between old white dwarfs (Han, Jeffery).

- Disks, Spots, Shells, Streams, Jets, ... What tends to make stars really unusual is not, normally, their internal structure, but what happens either at or above the surface - where we can see it best! Examples ranged from very young massive stars with vigorous outflows, through giants and degenerates around the fringes of the HR diagram, to microquasars in the stellar graveyard. Consider, for example, $\eta$ Car ("kinda like a volcano or geyser": Smith), $\epsilon$ Aur ("the mother of all accretion disks": Guinan), AG Dra ("as symbiotic as it gets": Wilson), and SS 433 (Chakrabarti).

Here also the big problems begin to manifest. Where does the UV iron emission forest come from in $\beta$ Lyr (Linnell)? Why have there been "over 900 papers [on $\gamma$ Cas] since 1930 , but few systematic or penetrative analyses" (Harmanec)? If we're lucky, we can resolve the structures that pose the problems: "There's one gorgeous picture of $\eta$ Car, there are lots of lovely pictures of $\beta$ Pic" (Lissauer).

Extended structures, generally disks, also provide the conditions necessary for objects like Nova Sco (Tout), high-luminosity Be stars (Miroschnichenko), and the possibility of forming icy planets alongside masers in the disks surrounding young stellar objects (Slysh, Val'tts).

- Geometry. If we can see it, then we have to solve the radiative transfer problem in order to understand what we see. This is hard enough in a one-dimensional plane-parallel semi-infinite geometry; it gets even harder in the real world. If nothing else, "exotic stars" emphasized the fact that few stars are simply single and spherical. That makes them interesting! In addition to the superficial material mentioned above, rotational distortion, Roche geometry, radiative distortion (Beer: not often considered before) and spots may all play a decisive rôle in governing stellar structure. Stellar distortions provoke various conundra: "it depends which end of the stars you're talking about, whether you mean the front end or the back end"; "I'm just trying to imagine a star living through this experience." Stars in which understanding the geometry represents a crucial step include $\eta \mathrm{Car}$ (obviously: Smith, Langer, Schöller), RY Sct (Smith), V471 Tau (Vaccaro, Guinan, Bond) and various HST planetary nebulae (Bond). But to make 
life really complicated, one should add a magnetic field which is never spherical and may do things that have not yet been discovered: "Next time you discover some peculiar variability, you might want to keep in mind that it could be one of these magnetically driven torques" (Lai).

- Is the Chemistry Working? Magnetic fields play another rôle in creating unusual stars by provoking chemical peculiarities. Amongst the Ap stars, for example, the prize for 'strangest star in class' was awarded to Przybylski's star ("don't claim to believe the observations unless you understand them": Kurtz), but HD 965, HD 217522 (Hubrig) and $o$ Leo (Griffin) received an honourable mention. HD 331319 provided the author with a mischievous thought; if $[\mathrm{He} / \mathrm{Fe}]=1.63$ (Miroshnichenko), what might $[\mathrm{Fe} / \mathrm{H}]$ be? Deficiencies in hydrogen are sometimes masked in cooler stars, but rarely in B stars such as BI Lyn (Jeffery), sole representative of several classes of extremely hydrogen-poor stars. Surprising as the misclassification of BILyn had been, it was harder to understand how the barium star WeBo 1 had originally been designated a planetary nebula nucleus (Bond). Nevertheless, it was a reminder that extreme chemical peculiarities are encountered throughout the HR diagram, and many have still to be understood.

- The Extreme, the Bizarre, the Surprising, the Deviant, the Wrecks. Despite the influence of binary interactions, stellar winds, and magnetic fields, most stars survive pretty well intact until they reach old age. The really strange objects are found in the mortuary. Consider, for example, SS 433 - "the most spectacular image in the X-ray sky" (Katani, Chakrabati) and the gargantuan carbon star CW Leo - "not a particularly weird or unusual star - but it does cover the largest extent on the sky" (Knapp). I was bewitched by the swiss-cheese character of the CW Leo stellar envelope indicated by submillimetre data; this seemed exotic enough to me, but why is it like that? Perhaps it had less to do with the star than with "the mess it's made of the ISM" (Knapp again). Born-again stars featured prominently, including FG Sge which (according to Schönberner \& Jeffery) is not H-deficient, and not particularly s-process rich. This group also includes V605 Aql, V4334 Sgr (Hinkle, Lawlor) and V838 Mon (Rauch), which had appeared just in time for the conference, and the naked white dwarf core $\mathrm{H} 1504+65$. Is the latter a rare O-Ne-Mg white dwarf (Werner)?

- Absent Friends Despite the number of quite extraordinary stars discussed, there were a considerable number of absentees. From a personal point of view, it would have been nice to have heard more about R CrB, V348 Sgr and other extremely hydrogen-poor relatives. Another missing VIP was the magnetic Bp star $\sigma$ Ori $\mathrm{E}$, a star with extreme $\mathrm{H} / \mathrm{He}$ anomalies across its surface and a prototype of the oblique rotator model. $v$ Sgr received a number of passing references, but deserved better, together with a host of evolved binaries (HD 128220, AA Dor, GP Com, PG 1336-018), pulsars, low-mass X-ray binaries, cataclysmic variables, and more besides. Sadly, we only had one week. 


\section{Solutions?}

Having gathered together all of the problems in stellar astronomy, the task ahead might seem daunting. However the conference suggested three broad approaches by which progress will undoubtedly be made in the near future. First among these is the acquisition of new data - although not necessarily to the liking of the delegate who suggested that "to understand stars better, we need less data, not more." Munari and $\mathrm{Zwitter}$ outlined prospective advances to be made with GAIA and "the grand armada of astrometric missions." Knowing distances and luminosities for every star in the galaxy will have a revolutionary impact. Griffin described a project for reclaiming the archives: stars work in the temporal domain, of which a substantial record is locked up in deteriorating photographic archives. The importance of other existing (INTEGRAL, Gimenez) and proposed (WSO/UV) missions was also touched on.

The second approach will be to develop better models. Three-dimensional stellar evolution with the Lawrence Livermore Djehuty code (Eggleton) promises much, but there is room for improvement in every field.

Finally, and a little tongue in cheek, one wonders whether the key to some exotic stars lies not in observing them ad infinitum but to choose a simpler but similar star or, in other words, to choose a simpler problem. Many problems are solved by breaking them up into smaller pieces and then joining up the results. It seems that some of the objects discussed this week, HR 2142 for example, might benefit from such a treatment.

\section{The truth is out there}

Although much of this conference has dwelt upon apparently insoluble problems in astronomy, there was much to be encouraged by. At the very worst, Eggleton remarked that "things in the heavens might not get quite as complex as things here on earth." Faced with an inundation of data from forthcoming projects, we shall be much exercised to identify what is exotic, "automating the identification of what is peculiar" (Kotani) will become a necessity, either to identify "another can of worms I don't want to get into" (Knapp), or to identify the really new exotic stars that will unlock the mysteries of the old. This leads me to reconsider our initial question - what makes a star exotic? I have deliberately echoed in the abstract what I offer here as a useful answer.

A star is exotic because it, in some way, extends our knowledge of how stars behave. As we make new observations we discover new phenomena that we do not understand. As we approach an understanding, those phenomena seem less exotic. That is nature. Our task is to keep the cycle of exploration, discovery and interpretation turning.

\section{Final Impressions}

In conclusion, IAU Colloquium 187 has left us with a set of impressions. The first is that of a family gathering. We renewed old acquaintances; some stars are just so exotic that they have defied explanation over the years. In the 
meantime, astronomy has turned up a number of new and equally unusual stars which challenge theories of stellar evolution.

The second was one of viewing a landscape. Listening to the talks and reading the posters we were presented with a series of snapshots of completely disparate objects, stellar evolution at all sorts of stages. Somehow they all lie in the same picture; our task is to figure out the connections.

We have carried out a a health check. There has been great progress in some areas of stellar astronomy. It was also evident that there has been comparatively little in others. This must be addressed.

For this reviewer there was a sense of a network in which there are ties between different types of star that may have been overlooked. It is hard to be specific, but often stars are classified by certain types of activity. Sometimes it is the stars we need to compare, and sometimes the activity.

There was clear evidence of a technology shortfall. It is time to translate the cartoons into 1-2-3d models with hydrodynamics, radiative transfer, etc. as required. This is no doubt hard, but only by understanding the geometry, and describing the system with robust physics, will we be able to answer many questions about stellar evolution.

Finally, I believe that this conference has provided a wake-up call. I was invigorated by learning something new about a lot of different stars. Stars are really interesting. They present an incredible range of properties, from the swisscheese structure of CW Leo to the enormous X-ray lobes of SS 433, from stellar wrecks like H 1504+065 to seemingly straightforward stars like our Sun.

The story of our science is a good story. We have a duty to tell it to our public. We should also pursue our science with confidence, using the fantastic facilities available to us today and coming online over the next decade.

Acknowledgments. The author is indebted to the International Astronomical Union and the Royal Society, London, for travel grants which made his attendance at IAU Colloquium 187 possible. All of the delegates are grateful to Bob Wilson and the scientific organizing committee and particularly to the real stars of the show, Walter van Hamme and his team of local organizers, for their efforts in making a memorable conference run smoothly. 